\title{
Endocannabinoid concentrations in hair are associated with PTSD symptom severity
}

\author{
Sarah Wilker ${ }^{\mathrm{a}, *}$, Anett Pfeiffer $^{\mathrm{b}}$, Thomas Elbert ${ }^{\mathrm{b}}$, Emilio Ovuga ${ }^{\mathrm{c}}$, Alexander Karabatsiakis ${ }^{\mathrm{a}}$, \\ Aniko Krumbholz ${ }^{\mathrm{d}}$, Detlef Thieme ${ }^{\mathrm{d}}$, Gustav Schelling ${ }^{\mathrm{e}}$, Iris-Tatjana Kolassa ${ }^{\mathrm{a}}$ \\ a Clinical \& Biological Psychology, Institute of Psychology \& Education, Ulm University, Albert-Einstein-Allee 47, 89069 Ulm, Germany \\ ${ }^{\mathrm{b}}$ Clinical Psychology, University of Konstanz, Universitätsstr. 10, 78457 Konstanz, Germany \\ ${ }^{\mathrm{c}}$ Faculty of Medicine, Gulu University, P.O. Box 166, Gulu, Uganda \\ d Institute of Doping Analysis and Sports Biochemistry Dresden, 01731 Kreischa, Germany \\ e Department of Anaesthesiology, Ludwig-Maximilians University, 82131 Munich, Germany
}

Keywords:

Posttraumatic stress disorder

Endocannabinoids

Hair

Palmitoylethanolamide

Oleoylethanolamine

Stearoylethanolamine

Mass spectrometry

\begin{abstract}
A B S T R A C T
The endocannabinoid system has been implicated in the regulation of the stress response, fear memory formation, and inflammatory processes. Posttraumatic stress disorder (PTSD) can result from exposure to extreme stress and is characterized by strong, associative memories for the traumatic events experienced. Furthermore, an elevated physical disease risk has been observed in PTSD, likely to be mediated by inflammatory processes. Therefore, altered endocannabinoid regulation can be expected in individuals with PTSD. However, attempts to assess PTSD-associated differences in the endocannabinoid system from human blood samples have provided inconsistent results, possibly due to fluctuating levels of endocannabinoids. In hair, these neuromodulators are accumulated over time and thus give access to a more stable and reliable assessment.

We therefore investigated PTSD-associated differences in hair concentrations of endocannabinoids $(\mathrm{N}$ acyl-ethanolamides palmitoylethanolamide [PEA], oleoylethanolamide [OEA] and stearoylethanolamide [SEA]) in 38 rebel war survivors from Northern Uganda suffering from PTSD and $N=38$ healthy rebel war survivors without current and lifetime PTSD. PTSD diagnosis and symptom severity were assessed in structured clinical interviews employing the Posttraumatic Diagnostic Scale (PDS). A significant group difference was observed for OEA, with PTSD patients showing reduced hair concentrations. Regression analyses further revealed strong negative relationships between all investigated $\mathrm{N}$-acyl-ethanolamides and symptom severity of PTSD. The observed reductions in endocannabinoids might account for the increased inflammatory state as well as for the failure to extinguish fear memories observed in PTSD. Our findings add to the accumulating evidence suggesting the endocannabinoid system as a target for pharmacological enhancement of exposure-based psychotherapy for PTSD.
\end{abstract}

\section{Introduction}

Posttraumatic stress disorder (PTSD) is a severe mental health disorder that can occur after extreme stress, such as war, rape or torture. Memory alterations represent the most characteristic feature of PTSD: While the traumatic events are re-experienced with high emotional and sensory intensity and vividness, the corresponding context information and chronological order is often difficult to recall (Brewin, 2015). In addition, PTSD is associated with an elevated risk for cardiovascular, autoimmune, metabolic

\footnotetext{
* Corresponding author

E-mail address: sarah.wilker@uni-ulm.de (S. Wilker).
}

and inflammatory diseases (Boscarino, 2004; Glaesmer et al., 2011; Roberts et al., 2015; Rosenbaum et al. 2015a; Seng et al., 2006).

The endocannabinoid system (ECS) comprises endogenous cannabinoid receptors and endocannabinoid neurotransmitters. The most extensive investigated endocannabinoids are anandamide (AEA) and 2-arachidonoylglycerol (2-AG) which both bind to $\mathrm{CB} 1$ and $\mathrm{CB} 2$ receptors. Other biologically highly active molecules linked to the ECS, which include $N$-acyl-ethanolamides such as palmitoylethanolamide (PEA), oleoylethanolamide (OEA) and stearoylethanolamide (SEA), are structurally highly similar to AEA and can potentiate its effects (Ho et al., 2008; Jonsson et al., 2001). Recently, PTSD research has focused on the ECS due to its role in stress regulation, memory processes, and inflammation (Neumeister et al., 2015). 
Animal and human research indicates a central role of endocannabinoids and related $\mathrm{N}$-acyl-ethanolamides in regulating the HPA axis and limiting stress reactions, as well as in the adaption to chronic stress. In humans, peripheral serum concentrations of the endogenous cannabinoid AEA and the related $\mathrm{N}$-acylethanolamides PEA and OEA were found to be elevated in the immediate aftermath of a social stressor (Dlugos et al., 2012), but declined in the subsequent recovery phase (Hill et al., 2009). Animal research has repeatedly shown a decrease in AEA in almost all brain regions investigated in response to both acute and chronic stress, which seems to contribute to HPA axis activation, as well as an anxiety phenotype (Morena et al., 2015). Furthermore, a peripheral decline of the $\mathrm{N}$-acylethanolamides PEA and OEA in the aftermath of predator stress was observed in animals, too (Holman et al., 2014). Correspondingly, knockout of endocannabinoid receptors was associated with impaired stress coping and enhanced anxiety and depressive-like symptoms, while stimulation of the ECS reduced anxiety and stress-related behavioral impairments in stress-exposed rats (reviewed in Hill and Patel (2013)).

Since PTSD is characterized by strong associative fear memories and a failure to extinguish fear responses to trauma reminders, fear conditioning research represents an important translational model for the disorder (Amstadter et al., 2009). Disruption of endocannabinoid signaling by peripheral administration of cannabinoid receptor antagonists or genetic knockout of cannabinoid receptors leads to impairment in extinction learning in animals (see Papini et al. (2015) for a systematic review). Furthermore, there is compelling evidence indicating that peripheral stimulation of the ECS e.g., by systemically administrating an inhibitor of fatty acid amide hydrolase, the most important enzyme for the degradation of AEA, PEA, OEA, and SEA, facilitates extinction learning and retention of extinction memory in both animals and humans (de Bitencourt et al., 2013; Papini et al., 2015).

Finally, endocannabinoids and related $N$-acyl-ethanolamides exert anti-inflammatory effects (Sayd et al., 2015). Taken together, the summarized literature strongly suggests that reduced endocannabinoid signaling in PTSD, a condition of chronic stress, could contribute to the explanation of the strong difficulties to extinguish fear reactions, the anxiety phenotype, as well as the state of low-grade inflammation observed in PTSD. Accordingly, the ECS has been proposed to play a significant role in PTSD etiology, and could represent a pharmacological treatment option for PTSD (Neumeister et al., 2015).

Indeed, reduced relative PEA concentrations were the strongest hallmark of PTSD in a metabolite profiling study (Karabatsiakis et al., 2015). Further studies also point towards reduced concentrations of some endocannabinoids and related $\mathrm{N}$-acyl-ethanolamides in PTSD (Hill et al., 2013; Neumeister et al., 2013). However, opposite or null effects have also been reported (Hauer et al., 2013; Schaefer et al., 2014). These inconsistencies might be due to strong variations of plasma endocannabinoid concentrations during the day (Vaughn et al., 2010). In particular in PTSD, recent intrusive symptoms might trigger the stress response and lead to daily variations in endocannabinoid concentrations.

The analyses of hair as opposed to blood, saliva or urine has long been used in toxicology, doping and forensic sciences. Substances (e.g., drugs of abuse or endogenous substances) can be incorporated into hair by different ways. Mainly discussed is the diffusion from the blood stream during formation of the hair shaft and the absorption after formation by sweat and sebum (Henderson, 1993). The advantages of hair analyses include high reliability, reduced bias by daily variations and the possibility of retrospective assessments over several months. The work group of Kirschbaum et al. (2009) introduced the idea that hair analyses might be useful to analyze the effects of psychological stress over longer time periods on stress hormones like glucocorticoids in human noninvasive studies. They were able to show that hair cortisol levels in pregnant women reflected the alterations of cortisol levels observed in peripheral blood during pregnancy (Kirschbaum et al., 2009). In a previous validation study, we observed that endocannabinoids tend to accumulate in hair during pregnancy (Krumbholz et al., 2013) and that this accumulation process can probably be compared to that seen with cortisol (Kirschbaum et al., 2009). The assessment of endocannabinoid concentrations in hair might hence represent a more reliable and stable assessment of the endocannabinoid system (Krumbholz et al., 2013) which could be especially useful for PTSD research.

Here, we present the results from the first study employing this novel method to investigate PTSD-associated endocannabinoid levels in hair samples of severely traumatized rebel war survivors from Northern Uganda. In line with the literature reviewed above, we hypothesized that hair concentrations of endocannabinoids/Nacyl-ethanolamides would be reduced in individuals with PTSD and correlate negatively with PTSD symptom severity.

\section{Material and methods}

\subsection{Study participants}

Analyses were based on a sample of $N=76$ survivors of the war between the rebel group Lord's Resistance Army (LRA) and the Ugandan Governmental forces from Northern Uganda. The recruitment took place in the former internal displaced people (IDP) camps Pabbo, Koch Goma and Pakiri in Northern Uganda. Initially, the study procedures were explained to the local leaders and community members in community meetings. During the data collection phase, trained local interviewers approached the clients in their homes, and invited one adult family member per household who was affected by the LRA conflict to participate. If more than one family member volunteered to participate, the most affected person was chosen, in order to recruit a sufficient number of current PTSD cases into the study.

The PTSD group consisted of $N=38$ LRA war survivors with a diagnosis of current PTSD according to DSM-IV, and a current symptom score $\geq 10$ who had never received any psychotherapeutic or medical treatment for their PTSD symptoms at the time of investigation. The control group comprised $N=38$ war survivors with the absence of a current, as well as a lifetime PTSD diagnosis. The groups were matched with respect to age and gender. Since both groups were recruited in the same former IDP camps, they faced similar living conditions. At the time of the assessment, the former camps were safe as rebel attacks within Northern Uganda had stopped since the ceasefire agreement in 2005. However, both groups faced daily stressors, which included poverty, and insufficient access to education or medical care.

\subsection{Clinical assessment}

Trained local interviewers performed the diagnostic interviews under the supervision of clinical psychologists specialized in traumatic stress studies from the universities of Ulm and Konstanz, Germany. The interviewers attended a six-week in-depth training on the concepts of quantitative data collection and mental health disorders focusing on the clinical assessment of traumatic event exposure and PTSD. All study instruments were translated into the local language, Luo. Translations were followed by blind backtranslations, and group discussions with independent interpreters, to ensure a valid translation of the instruments.

A 62-item event-list was employed to assess the number of traumatic event types experienced. This event-list was based on previous investigations of our work group in Sub-Saharan Africa 
(Kolassa et al., 2010a,b,c) and included events regarding general traumatic experiences (e.g., accidents, natural disasters), sexual and gender-based violence (e.g., rape, forced marriage, forced circumcision), war-related events (e.g., cross-fires, bombings) as well as child maltreatment (e.g., severe beatings by a caretaker). This event-list was further extended to include events specific for the atrocities committed by the LRA (e.g., being forced to eat human flesh, being forced to kill people). Accordingly, the resulting eventlist represented a very detailed record of traumatic events and encompassed 62 potentially traumatic events. For each event, participants were first asked, whether this event ever happened to them. We calculated the number of traumatic event types experienced as the number of the affirmative answers. This measure has been shown to be a reliable, valid and time-effective assessment of trauma exposure (Wilker et al., 2015). This event-list was assessed for the entire lifespan as well as for adverse experiences in the past year to assess current stressors.

The Posttraumatic Diagnostic Scale (PDS, Foa, 1995) was employed to assess the diagnosis of current and lifetime PTSD according to the DSM-IV, as well as current PTSD symptom severity and the sum scores of the symptom categories intrusions, avoidance and hyperarousal. The reliability and validity (i.e., consistency with expert ratings) of the translated PDS has been assured in a prior investigation (Ertl et al., 2010). Furthermore, we assessed symptoms of anxiety and depression with the respective section of the Hopkins Symptom Checklist (HSCL-25, Derogatis et al., 1974) and the diagnosis of a major depression with the Mini-International Neuropsychiatric Interview (M.I.N.I., Sheehan et al., 1998). After a detailed explanation of the study protocol, participants gave written informed consent. All procedures followed the Declaration of Helsinki and were approved by the institutional review board of Gulu University, Uganda, and the Ugandan National Council for Science and Technology (UNCST).

\subsection{Endocannabinoid analyses}

The interviewers collected hair strands as near to the scalp as feasible from a posterior vertex position. Since most participants had very short hair lengths (minimal length $\sim 5 \mathrm{~mm}$ ), the total length of available hair was analyzed. In seven cases (hair length $>3 \mathrm{~cm})$ only the scalp-nearest hair segment $(0-3 \mathrm{~cm})$ was used. Hair samples were weighed (range: 3-110 mg; median: $13 \mathrm{mg}$ ) and put into vials for homogenization. The complete amount of hair was powdered using a homogenizer (FastPrep 24, MP Biomedicals, Solon, Ohio, USA). After methanol extraction $(1.5 \mathrm{~mL}$ methanol, ultrasonic bath, $50^{\circ} \mathrm{C}, 10 \mathrm{~h}$ ), the hair residues were hydrolyzed with $1.5 \mathrm{~mL} 0.5 \mathrm{~N} \mathrm{KOH}$-solution for $12 \mathrm{~h}$ at $60^{\circ} \mathrm{C}$ after addition of $250 \mathrm{ng}$ PEA-d4 as internal standard (IS). Both, the liquid phase of the $\mathrm{MeOH}$-extraction (reconstituted with water) and the $\mathrm{KOH}$-phase were cleaned up by a solid-phase extraction using the Aspec XL4 (Gilson, Middleton, USA) and Plexa BondElut SPE columns ( $3 \mathrm{~mL}, 60 \mathrm{mg}$, Agilent Technologies, Böblingen, Germany). The SPE columns were pre-conditioned with $3 \mathrm{~mL}$ methanol and $1 \mathrm{~mL}$ water. After loading, columns were washed with $2 \mathrm{~mL}$ methanol/2\% NH4OH-solution (40/60; v/v) and eluted with $2 \mathrm{~mL}$ methanol/water $(90 / 10 ; \mathrm{v} / \mathrm{v})$. Extracts from both SPE-runs were combined, evaporated and reconstituted with $50 \mu \mathrm{L}$ HPLC-buffer. For each sample, $5 \mu \mathrm{L}$ were injected. Calibration samples, using solvent calibration, were processed as described above in concentration ranges between $1 \mathrm{ng} / \mathrm{mg}$ and $50 \mathrm{ng} / \mathrm{mg}$ for PEA, OEA and SEA based on $20 \mathrm{mg}$ weighted hair sample. LC-MS/MS-technology (LC-system: Agilent Technologies 1200 series, Böblingen, Germany, mass spectrometer: Q-Trap 5500, AB Sciex, Darmstadt, Germany) was used for concentration measurement. Chromatographic separation was operated on a Hypersil Gold C18-column $(100 \times 2.1$ $\mathrm{mm}, 3 \mu \mathrm{m}$; ThermoFisher Scientific, Langerwehe, Germany) protected by a Zorbax Eclipse XDB-C8-fingertight guard cartridge ( $2.1 \times 12.5 \mathrm{~mm}, 5 \mu \mathrm{m}$; Agilent Technologies, Böblingen, Germany). For identification and quantification two specific multiple reaction monitoring (MRM) transitions were optimized for all analytes (OEA: $326 \rightarrow 55 / 62$; PEA: $300 \rightarrow 283 / 62$; SEA: $328 \rightarrow 57 / 62$ ). The detected endocannabinoid levels were standardized to the total amount (mg) of vaporized hair.

The diagnostic value of endocannabinoids in hair has been validated in our previous investigation. Krumbholz et al. (2013) demonstrated that endocannabinoids are stable in different hair segments over time. Whereas hair cortisol concentrations declined by $50 \%$ over 4 months of hair growth, almost no wash-out effects on AEA, PEA, SEA and OEA levels were detectable. Furthermore, biochemical information provided by proximal hair segment analysis had face validity. Hair glucocorticoids and hair AEA showed negative correlations (Krumbholz et al., 2013), a relationship which has also been described in acutely stressed volunteers during a parabolic flight experiment (Chouker et al., 2010) and is in accordance with previously discussed regulatory effects of endocannabinoids on HPA-axis activation during chronic stress (Hill and McEwen, 2010).

\subsection{Statistical analyses}

\subsubsection{Analyses of demographic data, trauma exposure and} mental health

Demographic and clinical variables were compared between PTSD cases and controls using $t$-tests for continuous data, if model residuals were normally distributed, and the corresponding nonparametric test (Mann-Whitney- $U$-test), if the residuals were non-normally distributed. Categorical data was analyzed by $X^{2}$ tests and Fisher's exact tests, if the assumptions of the $X^{2}$-test were violated. The reported statistical tests are two-tailed, the significance level was set at $p \leq .05$.

\subsubsection{Analyses of endocannabinoid data}

Due to a skewed distribution of the endocannabinoid concentrations, endocannabinoid data was log-transformed prior to statistical analyses. In order to test the hypothesis that PTSD patients display lower levels in the measured endocannabinoid concentrations than the control group, we employed $t$-tests, if model residuals were normally distributed and Mann-Whitney- $U$ tests, if the residuals were non-normally distributed. To further test the hypothesis of a negative association between endocannabinoid concentrations and PTSD symptom severity, we calculated linear regression models with endocannabinoid concentration as the outcome variable and PTSD symptom severity as the predictor. We further investigated if the hypothesized negative association between endocannabinoid concentrations were also reflected in the PTSD symptoms scores of intrusions, avoidance and hyperarousal. To account for potential gender effects, we repeated the regression analyses for males and females separately. Finally, we investigated the relationship between endocannabinoid levels and lifetime trauma exposure, abduction time, and adversity in the past year.

All reported $p$-values are two-tailed, with a significance level set at $p \leq 0.05$. Since PEA, OEA and SEA levels were highly correlated $(r$ PEA,OEA $=.94, r_{\text {PEA,SEA }}=0.77, r_{\text {OEA,SEA }}=0.76$; all $\left.p<0.000001\right)$, similar effects could be expected for all three investigated endocannabinoids. Accordingly, the statistical tests were not independent from each other and a multiple comparison correction procedure would have been far too conservative. We therefore performed permutation tests of the $p$-values using 10,000 random permutations in order to account for any potential bias induced by multiple testing for the group comparisons, as well as the regression analyses with PTSD symptom severity and intrusions, avoidance and 
Table 1

Demographic and Clinical Data for PTSD Cases and Controls.

\begin{tabular}{|c|c|c|c|c|}
\hline Variable & Controls $(N=38)$ & $\operatorname{PTSD}(N=38)$ & Statistic ${ }^{a}$ & $p$ \\
\hline \multicolumn{5}{|l|}{ Demographics } \\
\hline Mean age $[S D]$ & $31[10.31]$ & 30.89 [9.13] & $W=680.5$ & 0.82 \\
\hline$N$ female $(\%)$ & $17(45 \%)$ & $20(53 \%)$ & $X^{2}=0.21$ & 0.65 \\
\hline Days of alcohol consumption & $4.45[9.07]$ & $2.21[5.56]$ & $W=736.5$ & 0.86 \\
\hline \multicolumn{5}{|l|}{ Trauma exposure } \\
\hline$N$ abducted $^{\mathrm{b}}(\%)$ & $17(45 \%)$ & $37(97 \%)$ & $X^{2}=23.09$ & $<0.0001$ \\
\hline Mean abduction time in months ${ }^{\mathrm{b}}[\mathrm{SD}]$ & $1.11[4.00]$ & $29.56[46.73]$ & $W=128$ & $<0.0001$ \\
\hline Mean number of lifetime traumatic event types experienced [SD] & $17.18[5.20]$ & 36.34 [6.09] & $t_{(\mathrm{df}=74)}=14.75$ & $<0.0001$ \\
\hline Mean number of traumatic event types experienced in the past year [SD] & $1.32[1.73]$ & $3.03[3.38]$ & $W=463.5$ & 0.006 \\
\hline Mean number of years since worst traumatic event [SD] & $10.84[6.6]$ & $12.11[5.18]$ & $t_{(\mathrm{df}=73)}=0.93$ & 0.36 \\
\hline \multicolumn{5}{|l|}{ Childhood traumatic experiences } \\
\hline$N$ severely hit by caretaker (\%) & $14(37 \%)$ & $17(45 \%)$ & $X^{2}=0.22$ & 0.64 \\
\hline$N$ burnt by caretaker $(\%)$ & $1(3 \%)$ & $5(13 \%)$ & Fisher's exact test & 0.20 \\
\hline \multicolumn{5}{|l|}{ Mental health symptoms } \\
\hline Mean PDS sum score [SD] & $1.32[2.01]$ & $18.32[5.64]$ & $W=0$ & $<0.0001$ \\
\hline Mean PDS sum score intrusions [SD] & $0.26[0.64]$ & $5.32[2.62]$ & $W=15.5$ & $<0.0001$ \\
\hline Mean PDS sum score avoidance [SD] & $0.58[0.92]$ & $6.53[2.63]$ & $W=2$ & $<0.0001$ \\
\hline Mean PDS sum score hyperarousal [SD] & $0.47[0.92]$ & $6.47[2.42]$ & $W=5$ & $<0.0001$ \\
\hline$N$ diagnosis of depressive episode (\%) & $0(0 \%)$ & $7(18 \%)$ & Fisher's exact test & 0.01 \\
\hline Mean HSCL depression score [SD] & $1.21[0.28]$ & $2.31[0.87]$ & $W=152.5$ & $<0.0001$ \\
\hline Mean HSCL anxiety score [SD] & $1.22[0.3]$ & $2.16[0.82]$ & $W=148.5$ & $<0.0001$ \\
\hline
\end{tabular}

PDS, Posttraumatic Diagnostic Scale, HSCL, Hopkins Symptom Checklist.

a Statistic refers to $X^{2}$-tests or Fisher's exact test for categorical data, and $t$-tests for continuous data if model residuals were normally distributed, Kruskal-Wallis $H$ test if residuals were non-normally distributed. All reported $p$-values are two-tailed tests.

b These demographic variables refer to abductions by the rebel group Lord's Resistance Army, generally associated with a high number of traumatic experiences.

Table 2

Association of endocannabinoid levels with PTSD symptomatology and trauma exposure.

\begin{tabular}{|c|c|c|c|c|}
\hline & $\beta$ & $\mathrm{SE}(\beta)$ & $t$-value & $p$ \\
\hline \multicolumn{5}{|l|}{ Prediction of PEA concentration } \\
\hline PDS sum score & -0.33 & 0.11 & 3.04 & 0.003 \\
\hline PDS sum score intrusions & -0.30 & 0.11 & 2.67 & 0.009 \\
\hline PDS sum score avoidance & -0.31 & 0.11 & 2.81 & 0.006 \\
\hline PDS sum score hyperarousal & -0.32 & 0.11 & 2.89 & 0.005 \\
\hline Abduction time in months & -0.11 & 0.12 & 0.96 & 0.34 \\
\hline Number of lifetime traumatic event types experienced & -0.26 & 0.11 & 2.31 & 0.02 \\
\hline Number of traumatic event types experienced in the past year & -0.10 & 0.12 & 0.85 & 0.40 \\
\hline \multicolumn{5}{|l|}{ Prediction of OEA concentration } \\
\hline PDS sum score & -0.34 & 0.11 & 3.16 & 0.002 \\
\hline PDS sum score intrusions & -0.27 & 0.11 & 2.43 & 0.02 \\
\hline PDS sum score avoidance & -0.33 & 0.11 & 3.05 & 0.003 \\
\hline PDS sum score hyperarousal & -0.35 & 0.11 & 3.19 & 0.002 \\
\hline Abduction time in months & -0.07 & 0.12 & 0.58 & 0.56 \\
\hline Number of lifetime traumatic event types experienced & -0.28 & 0.11 & 2.55 & 0.01 \\
\hline Number of traumatic event types experienced in the past year & -0.08 & 0.12 & 0.65 & 0.52 \\
\hline \multicolumn{5}{|l|}{ Prediction of SEA concentration } \\
\hline PDS sum score & -0.27 & 0.11 & 2.35 & 0.02 \\
\hline PDS sum score intrusions & -0.20 & 0.11 & 1.77 & 0.08 \\
\hline PDS sum score avoidance & -0.27 & 0.11 & 2.41 & 0.02 \\
\hline PDS sum score hyperarousal & -0.26 & 0.11 & 2.29 & 0.03 \\
\hline Abduction time in months & -0.20 & 0.11 & 1.77 & 0.08 \\
\hline Number of lifetime traumatic event types experienced & -0.20 & 0.12 & 1.72 & 0.09 \\
\hline Number of traumatic event types experienced in the past year & -0.16 & 0.11 & 1.43 & 0.16 \\
\hline
\end{tabular}

Displayed are the results of single linear regression models with the logarithmized endocannabinoid levels as the outcome variable and the respective psychological variables as predictors. All reported $p$-values are two-tailed tests.

Symbols and abbreviations: $\beta$, standardized regression coefficient; SE, standard error of measurement, PDS, Posttraumatic Diagnostic Scale.

hyperarousal. Since permutated $p$-values did not differ from ordinary $p$-values, ordinary $p$-values are reported for all analyses.

\section{Results}

\subsection{Demographic data, trauma exposure and mental health}

As intended by the matching procedure, PTSD patients and controls did not differ regarding age and gender distribution. Furthermore, no group difference regarding reported alcohol consumption was found. PTSD patients had experienced higher levels of traumatic stress as indicated by a higher number of abductions by the LRA, longer abduction durations and a higher number of traumatic event types experienced. PTSD patients also reported a higher level of potentially traumatic experiences in the past year. The time since the worst traumatic experience as well as the frequency of childhood traumatic experiences did not differ between the diagnostic groups. Regarding mental health symptoms, PTSD patients presented with a higher symptom load of PTSD, as reflected in the total PDS sum score, as well as in the symptom scores of 

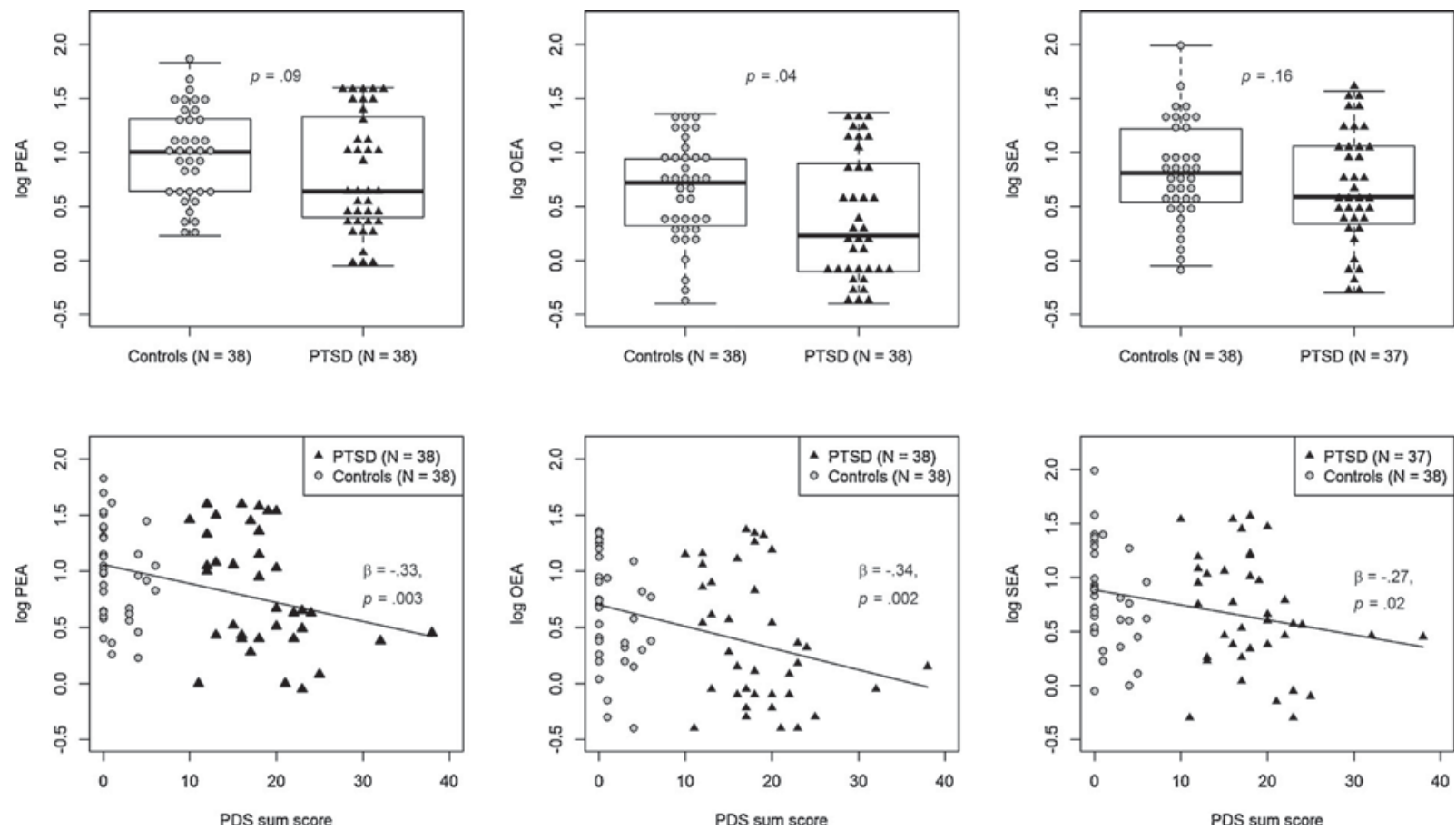

Fig. 1. Upper Panel: Box plots and raw values of logarithmized $N$-acyl-ethanolamides concentrations in hair by PTSD group. $p, p$-value obtained from $t$-tests, if test residuals were normally distributed, and Mann-Whitney-U-tests if the residuals were non-normally distributed. Lower Panel: Logarithmized $N$-acyl-ethanolamides are plotted against PTSD symptom severity. Displayed are the results of linear regression models with logarithmized $N$-acyl-ethanolamides as the dependent variables and PTSD symptom severity as the predictor. PDS, Posttraumatic Diagnostic Scale; $\beta$, standardized regression coefficient; $p$, $p$-value obtained from the significance test of the $\beta$-regression coefficient.
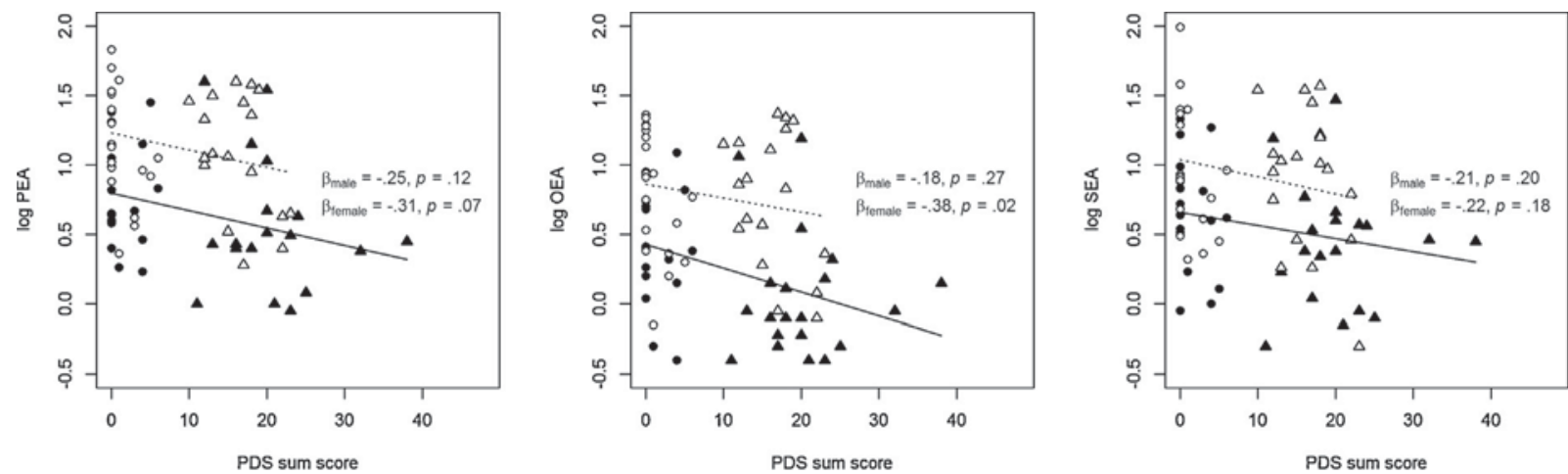

$\begin{array}{lllll}- \text { Controls female } & \Delta & \text { PTSD female } & - & \text { Regression female } \\ \circ \text { Controls male } & \Delta & \text { PTSD male } & \cdots \cdots & \text { Regression male }\end{array}$

Fig. 2. Logarithmized $N$-acyl-ethanolamides are plotted against PTSD symptom severity. Displayed are the results of linear regression models with logarithmized $N$-acylethanolamides as the dependent variables and PTSD symptom severity as the predictor conducted separately for males and females. PDS, Posttraumatic Diagnostic Scale; $\beta$, standardized regression coefficient; $p$, $p$-value obtained from the significance test of the $\beta$-regression coefficient.

intrusions, avoidance and hyperarousal. Furthermore, symptoms of anxiety and depression were more pronounced in the PTSD group. However, only 7 PTSD patients fulfilled the diagnostic criteria of a major depression. Table 1 presents demographic information, trauma exposure and mental health symptoms for PTSD patients and controls.

\subsection{Endocannabinoid data}

The group comparisons revealed a significantly lower concentration of OEA $\left(t_{\mathrm{df}}=74=2.09, p=.04\right.$, Fig. 1$)$ in PTSD patients as opposed to controls. A trend for a lower PEA concentration in PTSD was also found ( $W=884, p=.09$, Fig. 1 ), while SEA concentrations did not differ significantly between groups $\left(t_{\mathrm{df}}=73=1.40, p=.16\right.$, Fig. 1).

Yet, the associations between PTSD symptom severity and endocannabinoid concentrations were more pronounced. In linear regression models, we observed strong negative relationships between PTSD symptom severity and the respective endocannabinoid concentrations (PEA, $\beta=-.33, p=.003$; OEA, $\beta=-.34, p=.002$; SEA, $\beta=-.27, p=.02$; see Table 2 and Fig. 1 ). Women generally displayed significantly lower hair concentrations of PEA, SEA and OEA then men (all $p<.01$ ), and a trend for higher PTSD symptoms $(p=.11)$. When analyzing females and males separately, negative associations between PTSD symptom severity and endocannabinoid levels were observed in both groups, however, these 

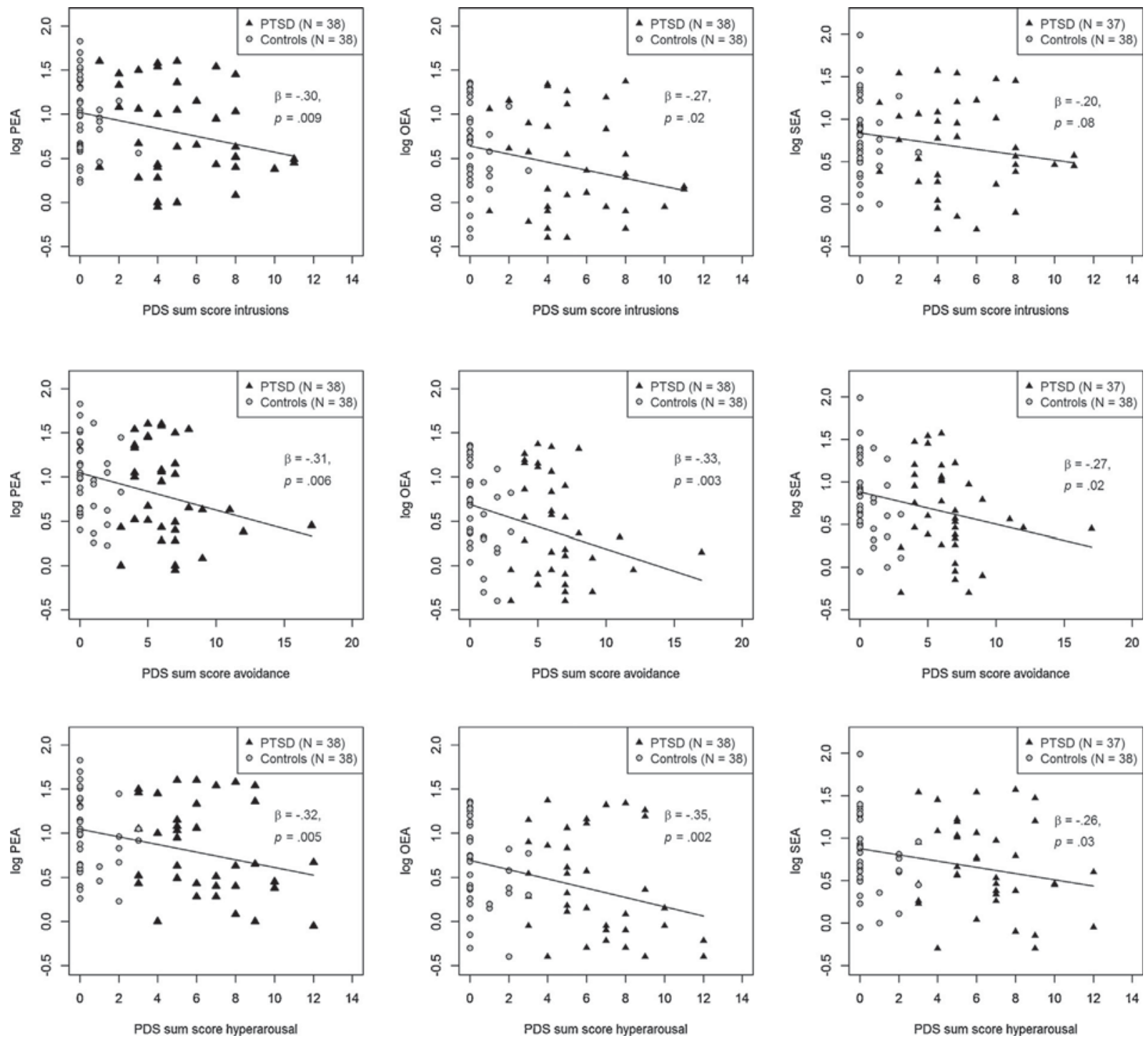

Fig. 3. Logarithmized $N$-acyl-ethanolamides are plotted against the symptom scores of intrusions, avoidance and hyperarousal. Displayed are the results of linear regression models with logarithmized $\mathrm{N}$-acyl-ethanolamides as the dependent variables and the respective PTSD symptom score as the predictor. PDS, Posttraumatic Diagnostic Scale; $\beta$, standardized regression coefficient; $p$, $p$-value obtained from the significance test of the $\beta$-regression coefficient.

associations did not always reach statistical significance when analyzing the subsamples of female and male participants (Females: PEA, $\beta=-.31, p=.07$; OEA, $\beta=-.38, p=.02$; SEA, $\beta=-.22, p=.18$; Males: PEA, $\beta=-.25, p=.12$; OEA, $\beta=-.18, p=.27$; SEA, $\beta=-.21$, $p=.20$; see Supplementary Tables 1 and 2 and Fig. 2 ).

The negative relationship between PTSD symptom severity and endocannabinoid levels was also reflected in strong negative associations between the symptom scores intrusions, avoidance and hyperarousal and PEA, OEA and SEA concentrations (see Table 2 and Fig. 3). Separate analyses of these associations for males and females also showed negative relationships, but did mostly not reach statistical significance (see Supplementary Tables 1 and 2 and Supplementary Fig. 1).

In order to investigate the effect of cumulative trauma exposure on endocannabinoid concentrations, we also investigated the association of the number of lifetime traumatic event types experienced and PEA, SEA and OEA concentrations in regression models accounting for PTSD group. In linear regression models, we also observed a negative relationship between lifetime trauma exposure and the endocannabinoid concentrations, however the association was weaker compared to PTSD symptom severity (see
Table 2). Regression models investigating the relationship between the duration of the abduction and endocannabinoid concentrations generally pointed towards the same direction but did not reach statistical significance (see Table 2). Finally, we did not observe any association between potentially traumatic experiences in the past year and endocannabinoid concentrations (see Table 2).

\section{Discussion}

Results reveal an association between PTSD and reduced hair concentrations of the $\mathrm{N}$-acyl-ethanolamides PEA, OEA and SEA. While the distributions of the diagnostic groups overlapped, we observed strong negative relationships between PTSD symptom severity and $N$-acyl-ethanolamides: The higher the PTSD symptoms, the lower the concentrations of PEA, OEA and SEA. Similar strong negative associations were also found for the symptom scores of intrusions, avoidance and hyperarousal, indicating a robust negative relationship between endocannabinoid concentrations and PTSD symptomatology across all symptom clusters. A separate analysis of female and male subjects also revealed similar negative associations for both sexes; however, results did not 
reach statistical significance in most cases, which is likely due to the reduced sample size and power when analyzing the smaller subsamples of males and females.

Several explanations might account for the negative association between PTSD symptomatology and endocannabinoid levels: First of all, the chronic stress associated with PTSD psychopathology (including symptoms of upsetting memories, nightmares, flashbacks, lack of sleep, a permanent state of alertness, and reduced psychosocial functioning) might lead the observed reductions in endocannabinoid levels. Second, repeated exposure to traumatic experiences might lead to differences in endocannabinoid levels. Indeed, we also observed negative relationships with the number of lifetime traumatic experiences and abduction time (Table 2); however, these effects were less pronounced compared to the effects of PTSD symptoms, indicating that the stress caused by severe PTSD symptoms might lead to alterations in endocannabinoid signaling above and beyond the effect of trauma exposure. Yet, due to the strong dose-response relationship between trauma exposure and PTSD symptom severity (Kolassa et al., 2010b; Wilker et al., 2015), it is hardly possible to disentangle the effects of trauma exposure and PTSD symptomatology. An alternative explanation might also be that pre-existing differences in endocannabinoid signaling influence the individual vulnerability to PTSD development. While these effects cannot be excluded, the association between trauma exposure and endocannabinoid levels illustrates the strong influence of environmental exposure. Finally, one might also think that our findings could be due to the higher number of current traumatic experiences in individuals with PTSD, however, this explanation can be ruled out as no association between current adverse experiences and hair concentrations of PEA, OEA and SEA was found.

Our results are in accordance with the stress literature indicating reductions in AEA and related $N$-acyl-ethanolamides levels in the aftermath of stress observed in peripheral blood in humans (Hill et al., 2009), as well as in different brain regions in animals (Morena et al., 2015), which seem to be associated with impairments in stress-coping and anxiety and depression symptoms (Hill and Patel, 2013).

Furthermore, the observed associations correspond well with findings suggesting that PTSD symptom severity is associated with enhanced risk for physical disease (Roberts et al., 2015). One reason for this enhanced disease susceptibility might be the state of low-grade inflammation observed in PTSD (Baker et al., 2012). In more detail, the spontaneous production (Gola et al., 2013) as well as the plasma levels of pro-inflammatory cytokines (von Kanel et al., 2007) were found to correlate positively with symptom severity of PTSD. OEA and PEA are known to inhibit inflammation by binding type-alpha peroxisome proliferator-activated receptors (PPAR-alpha), thus in the presence of a reduction in $\mathrm{N}$ acyl-ethanolamide signaling, inflammation may be increased. OEA is also released when dietary fat enters the small intestine where it serves as a mediator of fat-induced satiety (Rodriguez de Fonseca et al., 2001), regulates feeding and body weight and stimulates fat utilization (O'Sullivan and Kendall, 2010; Thabuis et al., 2008). The intraperitoneal administration of OEA to obese Zucker rats reduced food intake, lowered body weight, reduced hyperlipidemia and increased the transcription of PPAR-alpha (Fu et al., 2005). The risk of the metabolic syndrome and the associated cardiovascular complications in patients with PTSD is almost double as high as for the general population (Rosenbaum et al., 2015a). Recent crosssectional and long-term follow-up studies in patients with PTSD have clearly shown that indicators of the metabolic syndrome and the associated systemic inflammation increased with PTSD severity (Farr et al., 2015).

Individuals with PTSD also showed a poorer diet quality with a higher consumption of trans-fatty acids in traumatized individuals (Gavrieli et al., 2015). In our study, hair concentrations of
OEA decreased significantly with increased PTSD symptom intensity which suggests that impairments in OEA signaling could be a mediating factor in the increased prevalence of fat consumption, obesity, insulin resistance and cardiovascular risk commonly seen in patients with PTSD (Farr et al., 2015). Additional lifestyle factors, which could increase cardiovascular risk in individuals with PTSD, include a lack of physical activity (Gavrieli et al., 2015). Exercise has also been shown to improve symptoms in PTSD patients (Rosenbaum et al., 2015b). Interestingly, physical exercise increases peripheral endocannabinoid concentrations in humans (Feuerecker et al., 2012) and endocannabinoid receptors mediate anxiolysis and pain reduction in mice after exercise (Fuss et al., 2015). In sum, the negative, dose-dependent relationship between PTSD symptom severity and $\mathrm{N}$-acyl-ethanolamides might contribute to the explanation of the association between PTSD symptoms and inflammation, increased cardiovascular risk and the metabolic syndrome.

Given the central role of memory processes in the etiology, chronification and treatment of PTSD (Ehlers et al., 2004; Wilker and Kolassa, 2013; Wilker et al., 2014) and the role of the ECS in fear memory consolidation and extinction (de Bitencourt et al., 2013; Papini et al., 2015), our findings correspond with some of the memory alterations observed in PTSD. A large number of animal studies investigated the effects of blocking the ECS by either genetic knockout of cannabinoid receptors, or by peripheral administration of cannabinoid receptor antagonists and consistently found impairments in extinction learning (de Bitencourt et al., 2013; Papini et al., 2015). Correspondingly, pharmacological stimulation of the ECS by systemically administrating an inhibitor of fatty acid amide hydrolase, the most important enzyme for the degradation of AEA, PEA, OEA, and SEA, was associated with facilitation of extinction learning (Gunduz-Cinar et al., 2013; Papini et al., 2015). Interestingly, an increase in plasma OEA concentration as a result of intraperitoneal administration in rats has also been shown to enhance memory consolidation by engaging PPAR-alpha and recruiting local afferents of the vagal nerve in rats (Campolongo et al., 2009) and changes in peripheral OEA levels might therefore also influence extinction learning.

Furthermore, initial research in humans regarding exogenous stimulation of the ECS with THC prior to extinction learning indicated enhanced within-session fear memory extinction learning; however, this effect did not last until the retention test two days later (Klumpers et al., 2012). By contrast, Rabinak et al. (2013) observed enhanced extinction memory retention when THC was administered, but no immediate effects on extinction learning. This effect might be mediated by increased activity in the basolateral part of the amygdala, the prefrontal cortex and hippocampus during extinction memory recall, brain structures centrally involved in extinction learning (Rabinak et al., 2014). Although the small number of studies in humans as well as the inconsistent findings regarding immediate or prolonged effects of ECS stimulation warrant further investigation, these studies indicate that stimulation of the ECS might facilitate extinction learning in humans, too. Since exposure-based psychotherapies for PTSD rely on mechanisms of extinction learning (Anderson and Insel, 2006), it was argued whether stimulation of the ECS might augment therapeutic effects in PTSD (Neumeister et al., 2015). A preliminary randomized controlled trial indicated that treatment with a synthetic cannabinoid (mimicking the effects of THC) significantly reduces the reliving of the traumatic experiences in the form of nightmares in PTSD patients (Jetly et al., 2015). Similarly, a significant reduction in nightmares was observed, if THC was administered in addition to the ongoing medication in a preliminary, openlabel study (Roitman et al., 2014). While administration of THC in PTSD patients might be problematic due to the associated risk of addiction and abuse, potential cognitive impairments, as well the 
pleiotropic effectors of THC, these studies demonstrate that targeting the ECS might be a promising avenue for PTSD treatment research. A safer and more specific way to stimulate the ECS could be the administration of fatty acid amide hydrolase inhibitors to counteract endocannabinoid degradation (Neumeister et al., 2015; Papini et al., 2015) or specific inhibitors of $N$-acylethanolamine acid amidase, the metabolizing enzyme of PEA and OEA (Bandiera et al., 2014).

\section{Strength, limitations and future directions}

Strengths of the study include the relatively large sample size, the assessment of PTSD via structured clinical interviews and the recruiting of a relatively homogenous sample of participants who were all exposed to the LRA war. However, as indicated in the methods and in contrast to a previous study (Krumbholz et al., 2013), we were unable to reliably quantify the classical endocannabinoids 2AG and AEA in our hair samples. 2-AG and AEA concentrations in hair are extremely low, however, and the limited amount of hair available for analyses due to very short hair lengths of study participants may be one of the reasons why 2-AG and AEA measurements failed in our study. Furthermore, the cross-sectional design of our study not allow for a causal interpretation between PTSD symptoms and endocannabinoid hair concentrations.

Since this is the first study linking PTSD symptom severity to PEA, OEA and SEA concentrations in hair, the results of this study require replication. Future interesting research avenues might further include prospective studies in order to differentiate whether endocannabinoid alterations represent a risk factor for PTSD development or a consequence of the disorder. Last but not least, the role of endocannabinoids and related $\mathrm{N}$-acyl-ethanolamides in PTSD treatment requires further scientific attention. In particular, while some preliminary studies investigated the effects of pharmacological stimulation of the ECS in PTSD, research regarding the potential augmentation of exposure-based psychotherapy is lacking.

\section{Conflict of interest}

None.

\section{Contributors}

Sarah Wilker and Iris-Tatjana Kolassa conceptualized the study. Sarah Wilker and Anett Pfeiffer were responsible for the study setup and data acquisition in Uganda, with support from Thomas Elbert, Emilio Ovuga and Iris-Tatjana Kolassa. Alexander Karabatsiakis and Sarah Wilker prepared the hair samples for the endocannabinoid analyses. Gustav Schelling, Detlef Thieme and Aniko Krumbholz conducted the endocannabinoid analyses. Sarah Wilker conducted the statistical analyses and drafted the manuscript. All authors critically revised the manuscript and approved the final version of the manuscript.

\section{Role of the funding source}

We acknowledge the German National Academic Foundation (Studienstiftung des deutschen Volkes) for supporting this work through a scholarship awarded to Sarah Wilker.

\section{Acknowledgments}

We acknowledge the German National Academic Foundation (Studienstiftung des deutschen Volkes) for supporting this work through a scholarship awarded to Sarah Wilker. We would furthermore like to thank our team of Ugandan interviewers for conducting the interviews with exceptional commitment and empathy. Furthermore, we are thankful to Dr. Stephan Kolassa for valuable input regarding the statistic analyses.

\section{Appendix A. Supplementary data}

Supplementary data associated with this article can be found, in the online version, at http://dx.doi.org/10.1016/j.psyneuen.2016. 02.010 .

\section{References}

Amstadter, A.B., Nugent, N.R., Koenen, K.C., 2009. Genetics of PTSD: fear conditioning as a model for future research. Psychiatr. Ann. 39 (6), 358-367, http://dx.doi.org/10.3928/00485713-20090526-01.

Anderson, K.C., Insel, T.R., 2006. The promise of extinction research for the prevention and treatment of anxiety disorders. Biol. Psychiatry 60 (4), 319-321, http://dx.doi.org/10.1016/j.biopsych.2006.06.022.

Baker, D.G., Nievergelt, C.M. O'Connor, D.T, 2012. Biomarkers of PTSD: neuropeptides and immune signaling. Neuropharmacology 62 (2), 663-673, http://dx.doi.org/10.1016/j.neuropharm.2011.02.027.

Bandiera, T., Ponzano, S., Piomelli, D., 2014. Advances in the discovery of $\mathrm{N}$-acylethanolamine acid amidase inhibitors. Pharmacol. Res. 86, 11-17, http:// dx.doi.org/10.1016/j.phrs.2014.04.011.

Boscarino, J.A., 2004. Posttraumatic stress disorder and physical illness: results from clinical and epidemiologic studies. Ann. N. Y. Acad. Sci. 1032, 141-153, http://dx.doi.org/10.1196/annals.1314.011.

Brewin, C.R., 2015. Re-experiencing traumatic events in PTSD: new avenues in research on intrusive memories and flashbacks. Eur. J. Psychotraumatol. 6 , 27180, http://dx.doi.org/10.3402/ejpt.v6.27180.

Campolongo, P., Roozendaal, B., Trezza, V., Cuomo, V., Astarita, G., Fu, J., McGaugh, J.L., Piomelli, D., 2009. Fat-induced satiety factor oleoylethanolamide enhances memory consolidation. Proc. Natl. Acad. Sci. U. S. A. 106 (19), 8027-8031, http://dx.doi.org/10.1073/pnas.0903038106.

Chouker, A., Kaufmann, I., Kreth, S., Hauer, D., Feuerecker, M., Thieme, D., Vogeser, M., Thiel, M., Schelling, G., 2010. Motion sickness, stress and the endocannabinoid system. PLoS One 5 (5), e10752, http://dx.doi.org/10.1371/ journal.pone.0010752.

de Bitencourt, R.M., Pamplona, F.A., Takahashi, R.N., 2013. A current overview of cannabinoids and glucocorticoids in facilitating extinction of aversive memories: potential extinction enhancers. Neuropharmacology 64, 389-395, http://dx.doi.org/10.1016/j.neuropharm.2012.05.039.

Derogatis, L.R., Lipman, R.S., Rickels, K., Uhlenhuth, E.H., Covi, L., 1974. The hopkins symptom checklist: a self-report symptom inventory. Behav. Sci. 19, 1-15.

Dlugos, A., Childs, E., Stuhr, K.L., Hillard, C.J., de Wit, H., 2012. Acute stress increases circulating anandamide and other $N$-acylethanolamines in healthy humans. Neuropsychopharmacology 37 (11), 2416-2427, http://dx.doi.org/10.1038/ npp.2012.100.

Ehlers, A., Hackmann, A., Michael, T., 2004. Intrusive re-experiencing in post-traumatic stress disorder: phenomenology, theory, and therapy. Memory 12 (4), 403-415, http://dx.doi.org/10.1080/09658210444000025.

Ertl, V., Pfeiffer, A., Saile, R., Schauer, E., Elbert, T., Neuner, F., 2010. Validation of a mental health assessment in an african conflict population. Psychol. Assess. 22 (2), 318-324, http://dx.doi.org/10.1037/a0018810.

Farr, O.M., Ko, B.J., Joung, K.E., Zaichenko, L., Usher, N., Tsoukas, M., Thakkar, B., Davis, C.R., Crowell, J.A., Mantzoros, C.S., 2015. Posttraumatic stress disorder, alone or additively with early life adversity, is associated with obesity and cardiometabolic risk. Nutr. Metab. Cardiovasc. Dis.: NMCD 25 (5), 479-488, http://dx.doi.org/10.1016/j.numecd.2015.01.007.

Feuerecker, M., Hauer, D., Toth, R., Demetz, F., Holzl, J., Thiel, M., Kaufmann, I., Schelling, G., Chouker, A., 2012. Effects of exercise stress on the endocannabinoid system in humans under field conditions. Eur. J. Appl. Physiol. 112 (7), 2777-2781, http://dx.doi.org/10.1007/s00421-011-2237-0.

Foa, E.B., 1995. Posttraumatic Stress Diagnostic Scale Manual. National Computer Systems, Minneapolis.

Fu, J., Oveisi, F., Gaetani, S., Lin, E., Piomelli, D., 2005. Oleoylethanolamide, an endogenous PPAR-alpha agonist, lowers body weight and hyperlipidemia in obese rats. Neuropharmacology 48 (8), 1147-1153, S0028-3908(05)00089-4.

Fuss, J., Steinle, J., Bindila, L., Auer, M.K., Kirchherr, H., Lutz, B., Gass, P., 2015. A runner's high depends on cannabinoid receptors in mice. Proc. Natl. Acad. Sci. U. S. A. 112 (42), 13105-13108, http://dx.doi.org/10.1073/pnas.1514996112.

Gavrieli, A., Farr, O.M., Davis, C.R., Crowell, J.A., Mantzoros, C.S., 2015. Early life adversity and/or posttraumatic stress disorder severity are associated with poor diet quality, including consumption of trans fatty acids, and fewer hours of resting or sleeping in a US middle-aged population: a cross-sectional and prospective study. Metabolism 64 (11), 1597-1610, http://dx.doi.org/10.1016/ j.metabol.2015.08.017.

Glaesmer, H., Brahler, E., Gündel, H., Riedel-Heller, S.G., 2011. The association of traumatic experiences and posttraumatic stress disorder with physical morbidity in old age: a german population-based study. Psychosom. Med. 73 (5), 401-406, http://dx.doi.org/10.1097/PSY.0b013e31821b47e8.

Gola, H., Engler, H., Sommershof, A., Adenauer, H., Kolassa, S., Schedlowski, M., Groettrup, M., Elbert, T., Kolassa, I.T., 2013. Posttraumatic stress disorder is associated with an enhanced spontaneous production of pro-inflammatory cytokines by peripheral blood mononuclear cells. BMC Psychiatry 13, 40, http://dx.doi.org/10.1186/1471-244X-13-40.

Gunduz-Cinar, O., MacPherson, K.P., Cinar, R., Gamble-George, J., Sugden, K., Williams, B., Godlewski, G., Ramikie, T.S., Gorka, A.X., Alapafuja, S.O., Nikas, 
S.P. Makriyannis, A, Poulton, R, Patel, S., Hariri, A.R, Caspi, A, Moffitt, T.E. Kunos, G., Holmes, A., 2013. Convergent translational evidence of a role for anandamide in amygdala-mediated fear extinction, threat processing and stress-reactivity. Mol. Psychiatry 18 (7), 813-823, http://dx.doi.org/10.1038/ mp.2012.72.

Hauer, D., Schelling, G., Gola, H., Campolongo, P., Morath, J., Roozendaal, B., Humuni, G., Karabatsiakis, A., Atsak, P., Vogeser, M., Kolassa, I.T., 2013. Plasma concentrations of endocannabinoids and related primary fatty acid amides in patients with post-traumatic stress disorder. PLoS One 8 (5), e62741, http://dx. doi.org/10.1371/journal.pone.0062741.

Henderson, G.L., 1993. Mechanisms of drug incorporation into hair. Forensic Sci. Int. 63 (1-3), 19-29, 0379-0738(93) 90256-A.

Hill, M.N., Bierer, L.M., Makotkine, I., Golier, J.A., Galea, S., McEwen, B.S., Hillard, C.J., Yehuda, R., 2013. Reductions in circulating endocannabinoid levels in individuals with post-traumatic stress disorder following exposure to the world trade center attacks. Psychoneuroendocrinology 38 (12), 2952-2961, http://dx.doi.org/10.1016/j.psyneuen.2013.08.004.

Hill, M.N., McEwen, B.S., 2010. Involvement of the endocannabinoid system in the neurobehavioural effects of stress and glucocorticoids. Prog. Neuro-Psychopharmacol. Biol. Psychiatry 34 (5), 791-797, http://dx.doi.org/ 10.1016/j.pnpbp.2009.11.001.

Hill, M.N., Miller, G.E., Carrier, E.J., Gorzalka, B.B., Hillard, C.J., 2009. Circulating endocannabinoids and $\mathrm{N}$-acyl ethanolamines are differentially regulated in major depression and following exposure to social stress. Psychoneuroendocrinology 34 (8), 1257-1262, http://dx.doi.org/10.1016/j. psyneuen.2009.03.013.

Hill, M.N., Patel, S., 2013. Translational evidence for the involvement of the endocannabinoid system in stress-related psychiatric illnesses. Biol. Mood Anxiety Disord. 3 (1), 19, http://dx.doi.org/10.1186/2045-5380-3-19.

Ho, W.S., Barrett, D.A., Randall, M.D., 2008. 'Entourage' effects of $\mathrm{N}$-palmitoylethanolamide and $\mathrm{N}$-oleoylethanolamide on vasorelaxation to anandamide occur through TRPV1 receptors. Br. J. Pharmacol. 155 (6), 837-846, http://dx.doi.org/10.1038/bjp.2008.324.

Holman, E.A., Guijarro, A., Lim, J., Piomelli, D., 2014. Effects of acute stress on cardiac endocannabinoids, lipogenesis, and inflammation in rats. Psychosom. Med. 76 (1), 20-28, http://dx.doi.org/10.1097/PSY.0000000000000025.

Jetly, R., Heber, A., Fraser, G., Boisvert, D., 2015. The efficacy of nabilone, a synthetic cannabinoid, in the treatment of PTSD-associated nightmares: a preliminary randomized, double-blind, placebo-controlled cross-over design study. Psychoneuroendocrinology 51, 585-588, http://dx.doi.org/10.1016/j. psyneuen.2014.11.002.

Jonsson, K.O., Vandevoorde, S., Lambert, D.M., Tiger, G., Fowler, C.J., 2001. Effects of homologues and analogues of palmitoylethanolamide upon the inactivation of the endocannabinoid anandamide. Br. J. Pharmacol. 133 (8), 1263-1275, http://dx.doi.org/10.1038/sj.bjp.0704199.

Karabatsiakis, A., Hamuni, G., Wilker, S., Kolassa, S., Renu, D., Kadereit, S., Schauer, M., Hennessy, T., Kolassa, I.T., 2015. Metabolite profiling in posttraumatic stress disorder. J. Mol. Psychiatry 3 (1), 2, http://dx.doi.org/10.1186/s40303015-0007-3.

Kirschbaum, C., Tietze, A., Skoluda, N., Dettenborn, L., 2009. Hair as a retrospective calendar of cortisol production-increased cortisol incorporation into hair in the third trimester of pregnancy. Psychoneuroendocrinology 34 (1), 32-37, http:// dx.doi.org/10.1016/j.psyneuen.2008.08.024

Klumpers, F., Denys, D., Kenemans, J.L., Grillon, C., van der Aart, J., Baas, J.M., 2012. Testing the effects of delta9-THC and $D$-cycloserine on extinction of conditioned fear in humans. J. Psychopharmacol. 26 (4), 471-478, http://dx. doi.org/10.1177/0269881111431624.

Kolassa, I.T., Ertl, V., Eckart, C., Glockner, F., Kolassa, S., Papassotiropoulos, A., de Quervain, D.J., Elbert, T., 2010a. Association study of trauma load and SLC6A4 promoter polymorphism in posttraumatic stress disorder: evidence from survivors of the rwandan genocide. J. Clin. Psychiatry 71 (5), 543-547, http:/ dx.doi.org/10.4088/JCP.08m04787blu.

Kolassa, I.T., Ertl, V., Kolassa, S., Onyut, L.P., Elbert, T., 2010b. The probability of spontaneous remission from PTSD depends on the number of traumatic event types experienced. Psychol. Trauma 3 (3), 169-174, http://dx.doi.org/10.1037/ a0019362.

Kolassa, I.T., Kolassa, S., Ertl, V., Papassotiropoulos, A., De Quervain, D.J., 2010c. The risk of posttraumatic stress disorder after trauma depends on traumatic load and the catechol-o-methyltransferase val(158) met polymorphism. Biol. Psychiatry 67 (4), 304-308, http://dx.doi.org/10.1016/j.biopsych.2009.10.009.

Krumbholz, A., Anielski, P., Reisch, N., Schelling, G., Thieme, D., 2013. Diagnostic value of concentration profiles of glucocorticosteroids and endocannabinoids in hair. Ther. Drug Monit. 35 (5), 600-607, http://dx.doi.org/10.1097/FTD. 0b013e3182953e43.

Morena, M., Patel, S., Bains, J.S., Hill, M.N., 2015. Neurobiological interactions between stress and the endocannabinoid system. Neuropsychopharmacology, http://dx.doi.org/10.1038/npp.2015.166.

Neumeister, A., Normandin, M.D., Pietrzak, R.H., Piomelli, D., Zheng, M.Q., Gujarro-Anton, A., Potenza, M.N., Bailey, C.R., Lin, S.F., Najafzadeh, J., Ropchan, J., Henry, S., Corsi-Travali, S., Carson, R.E., Huang, Y., 2013. Elevated brain cannabinoid CB1 receptor availability in post-traumatic stress disorder: a positron emission tomography study. Mol. Psychiatry 18 (9), 1034-1040, http://dx.doi.org/10.1038/mp.2013.61.
Neumeister, A., Seidel, J., Ragen, B.J., Pietrzak, R.H., 2015. Translational evidence for a role of endocannabinoids in the etiology and treatment of posttraumatic stress disorder. Psychoneuroendocrinology 51, 577-584, http://dx.doi.org/10. 1016/j.psyneuen.2014.10.012.

O'Sullivan, S.E., Kendall, D.A., 2010. Cannabinoid activation of peroxisome proliferator-activated receptors: potential for modulation of inflammatory disease. Immunobiology 215 (8), 611-616, http://dx.doi.org/10.1016/j.imbio. 2009.09.007.

Papini, S., Sullivan, G.M., Hien, D.A., Shvil, E., Neria, Y., 2015. Toward a translational approach to targeting the endocannabinoid system in posttraumatic stress disorder: a critical review of preclinical research. Biol. Psychol. 104, 8-18, http://dx.doi.org/10.1016/j.biopsycho.2014.10.010.

Rabinak, C.A., Angstadt, M., Lyons, M., Mori, S., Milad, M.R., Liberzon, I., Phan, K.L. 2014. Cannabinoid modulation of prefrontal-limbic activation during fear extinction learning and recall in humans. Neurobiol. Learn. Mem. 113 125-134, http://dx.doi.org/10.1016/j.nlm.2013.09.009.

Rabinak, C.A., Angstadt, M., Sripada, C.S., Abelson, J.L., Liberzon, I., Milad, M.R., Phan, K.L., 2013. Cannabinoid facilitation of fear extinction memory recall in humans. Neuropharmacology 64, 396-402, http://dx.doi.org/10.1016/j. neuropharm.2012.06.063.

Roberts, A.L., Agnew-Blais, J.C., Spiegelman, D., Kubzansky, L.D., Mason, S.M., Galea, S., Hu, F.B., Rich-Edwards, J.W., Koenen, K.C., 2015. Posttraumatic stress disorder and incidence of type 2 diabetes mellitus in a sample of women: a 22-year longitudinal study. JAMA Psychiatry 72 (3), 203-210, http://dx.doi. org/10.1001/jamapsychiatry.2014.2632.

Rodriguez de Fonseca, F., Navarro, M., Gomez, R., Escuredo, L., Nava, F., Fu, J., Murillo-Rodriguez, E., Giuffrida, A., LoVerme, J., Gaetani, S., Kathuria, S., Gall, C., Piomelli, D., 2001. An anorexic lipid mediator regulated by feeding. Nature 414 (6860), 209-212, http://dx.doi.org/10.1038/35102582.

Roitman, P., Mechoulam, R., Cooper-Kazaz, R., Shalev, A., 2014. Preliminary, open-label, pilot study of add-on oral delta9-tetrahydrocannabinol in chronic post-traumatic stress disorder. Clin. Drug Invest. 34 (8), 587-591, http://dx. doi.org/10.1007/s40261-014-0212-3.

Rosenbaum, S., Stubbs, B., Ward, P.B., Steel, Z., Lederman, O., Vancampfort, D., 2015a. The prevalence and risk of metabolic syndrome and its components among people with posttraumatic stress disorder: a systematic review and meta-analysis. Metabolism 64 (8), 926-933, http://dx.doi.org/10.1016/j. metabol.2015.04.009.

Rosenbaum, S., Vancampfort, D., Steel, Z., Newby, J., Ward, P.B., Stubbs, B., 2015b. Physical activity in the treatment of post-traumatic stress disorder: a systematic review and meta-analysis. Psychiatry Res. 230 (2), 130-136, http:// dx.doi.org/10.1016/j.psychres.2015.10.017.

Sayd, A., Anton, M., Alen, F., Caso, J.R., Pavon, J., Leza, J.C., Rodriguez de Fonseca, F., Garcia-Bueno, B., Orio, L., 2015. Systemic administration of oleoylethanolamide protects from neuroinflammation and anhedonia induced by LPS in rats. Int. J. Neuropsychopharmacol. 18 (6), http://dx.doi.org/10.1093/ijnp/pyu111.

Schaefer, C., Enning, F., Mueller, J.K., Bumb, J.M., Rohleder, C., Odorfer, T.M. Klosterkotter, J., Hellmich, M., Koethe, D., Schmahl, C., Bohus, M., Leweke, F.M., 2014. Fatty acid ethanolamide levels are altered in borderline personality and complex posttraumatic stress disorders. Eur. Arch. Psychiatry Clin. Neurosci. 264 (5), 459-463, http://dx.doi.org/10.1007/s00406-013-0470-8.

Seng, J.S., Clark, M.K., McCarthy, A.M., Ronis, D.L., 2006. PTSD and physical comorbidity among women receiving medicaid: results from service-use data. J. Trauma Stress 19 (1), 45-56, http://dx.doi.org/10.1002/jts.20097.

Sheehan, D.V., Lecrubier, Y., Sheehan, K.H., Amorim, P., Janavs, J., Weiller, E., Hergueta, T., Baker, R., Dunbar, G.C., 1998. The mini-international neuropsychiatric interview (M.I.N.I.): the development and validation of a structured diagnostic psychiatric interview for DSM-IV and ICD-10. J. Clin. Psychiatry 59 (Suppl. 20), 22-33, quiz 34-57.

Thabuis, C., Tissot-Favre, D., Bezelgues, J.B., Martin, J.C., Cruz-Hernandez, C., Dionisi, F., Destaillats, F., 2008. Biological functions and metabolism of oleoylethanolamide. Lipids 43 (10), 887-894, http://dx.doi.org/10.1007/ s11745-008-3217-y.

Vaughn, L.K., Denning, G., Stuhr, K.L., de Wit, H., Hill, M.N., Hillard, C.J., 2010. Endocannabinoid signalling: Has it got rhythm? Br. J. Pharmacol. 160 (3), 530-543, http://dx.doi.org/10.1111/j.1476-5381.2010.00790.x.

von Kanel, R., Hepp, U., Kraemer, B., Traber, R., Keel, M., Mica, L., Schnyder, U., 2007. Evidence for low-grade systemic proinflammatory activity in patients with posttraumatic stress disorder. J. Psychiatr. Res. 41 (9), 744-752, http://dx.doi. org/10.1016/j.jpsychires.2006.06.009.

Wilker, S., Pfeiffer, A., Kolassa, S., Koslowski, D., Elbert, T., Kolassa, I.T, 2015. How to quantify exposure to traumatic stress? Reliability and predictive validity of measures for cumulative trauma exposure in a post-conflict population. Eur. J. Psychotraumatol. 6 (28306), http://dx.doi.org/10.3402/ejpt.v6.28306.

Wilker, S., Elbert, T., Kolassa, I.T., 2014. The downside of strong emotional memories: how human memory-related genes influence the risk for posttraumatic stress disorder-a selective review. Neurobiol. Learn. Mem. 112, 75-86, http://dx.doi.org/10.1016/j.nlm.2013.08.015.

Wilker, S., Kolassa, I.T., 2013. The formation of a neural fear network in posttraumatic stress disorder: insights from molecular genetics. Clin. Psychol. Sci. 1 (4), 452-469, http://dx.doi.org/10.1177/2167702613479583. 